\title{
Embryonic Beginnings of Definitive Hematopoietic Stem Cells
}

\author{
ELAINE DZIERZAK ${ }^{a}$ \\ Department of Cell Biology and Genetics, Medical Faculty, Erasmus University, \\ P.O. Box 1738, 3000 DR Rotterdam, The Netherlands
}

\begin{abstract}
The ability of the many cell types within the adult blood system to be constantly replenished and renewed from hematopoietic stem cells is an interesting problem in development and differentiation and has led to questions concerning how, when and where these stem cells for the adult hematopoietic system are generated within the embryo. During embryonic development many mature hematopoietic cells appear before adult-type hematopoietic stem cells thus the notion of a conventional hematopoietic hierarchy is challenged. Experiments probing the development of hematopoietic stem cells in the mouse embryo strongly suggest that at least two independent hematopoietic sites generate blood cells during development; the yolk sac, which produces the transient embryonic hematopoietic system, and the AGM (aorta-gonad-mesonephros) region, which initiates the long-lived adult hematopoietic system.
\end{abstract}

\section{INTRODUCTION}

The adult hematopoietic system is a complex series of pluripotent, multipotent and unipotent cellular intermediates which proliferate and differentiate into at least eight morphologically and functionally distinct mature blood cell types. Hematopoietic stem cells are at the foundation of this cellular hierarchy in the adult. The cells within this hierarchy have been defined by numerous in vitro and in vivo hematologic assays which measure proliferation and lineage differentiation. The most stringent of the assays, the radiation chimera assay, has been used to demonstrate the presence of pluripotential hematopoietic stem cells in bone marrow ${ }^{1}$ and fetal liver. ${ }^{14}$ In vivo transplantation of true adult-type hematopoietic stem cells into adult recipient mice depleted of endogenous hematopoietic stem cells by a lethal dose of irradiation leads to the complete, long-term engraftment of all blood lineages by donor-derived stem cells. Thus, pluripotential hematopoietic stem cells possess the following complex characteristics: 1) potential for all hematopoietic lineages as demonstrated by clonal markers; 2) high proliferative potential leading to $100 \%$ donor-derived engraftment; 3) long-term activity throughout the lifespan of the individual; and 4) self-renewal as demonstrated by in vivo serial transplantations. The clinical importance of stem cells with these characteristics is widely recognized in transplantation scenarios for blood-related genetic deficiencies and leukemias.

The developmental origins of adult-type hematopoietic stem cells in mammals are of current interest. ${ }^{6,7}$ In developmentally early hematopoietic microenviron-

${ }^{a}$ Phone, +31-10-408-7172; fax, +31-10-436-0225; e-mail, dzierzak@ch1.fgg.eur.nl 
ments, stem cells are induced and/or expanded from cells derived from the mesodermal germ layer. Subsequently, hematopoietic stem cells colonize the liver during fetal stages and the bone marrow of the adult where they remain throughout life..$^{13}$ Early hematopoietic development has been examined in both mammalian ${ }^{19}$ and nonmammalian ${ }^{4,5,24,25}$ vertebrate embryos. Mammalian vertebrates have the advantage of many in vitro and in vivo assays for defining the presence of an array of hematopoietic progenitors and stem cells within the embryo. Primitive erythroid cells, erythroid-myeloid progenitors, CFU-S and hematopoietic stem cells have been found in the yolk sac at E7, E8, E8.5, and E11, respectively. ${ }^{19}$ These results led to the previously accepted notion that the yolk sac was the source of the adult hematopoietic system in mammals. However, orthotopic grafting experiments which can be performed with the large embryos of avian ${ }^{4}$ or amphibian species ${ }^{25}$ showed that while the yolk sac or ventral blood islands (yolk sac analogue) produced hematopoietic cells, these were transient blood cells present predominantly during embryonic stages. The long-lived adult hematopoietic system was generated instead by the cells in an intraembryonic site surrounding the dorsal aorta and pro/mesonephros. The dichotomy in these results of higher and lower vertebrates persisted for over twenty years leading to the assumption that mammalian hematopoietic development is different from that in nonmammalian vertebrate species. With the absence of previous studies examining this intraembryonic site in mammals, we set out to determine whether hematopoietic activity was present and could take its origins in the region comprising the dorsal aorta, gonads and mesonephros (AGM) of the early and mid-gestation mouse embryo.

\section{THE AGM REGION CONTAINS HEMATOPOIETIC PROGENITORS} AND STEM CELLS

To examine the hematopoietic potential of the intraembryonic AGM region we first performed the short-term in vivo spleen colony-forming unit (CFU-S) assay. We injected a single cell suspension of various tissues from E8, E9, E10, or E11 mouse embryos into lethally irradiated adult female recipient mice and examined their spleens at 9 and 11 days posttransplantation for the presence of macroscopic (erythroid-myeloid) colonies. ${ }^{17}$ The donor injected cells were obtained from male embryos or transgenic embryos marked by either a Y chromosome or a human beta-globin gene locus (line 72), respectively. While E8 yolk sac, AGM, liver and blood contained no CFU-S, such progenitors are present in both E9 yolk sac and AGM. At E10 the number and frequency of CFU-S in the AGM region surpasses that in the yolk sac and peaks at late E10. Thereafter, CFU-S decrease rapidly in the AGM, with a concomitant increase in CFU-S in the liver. These results demonstrate the presence of potent multilineage progenitors in the AGM region, and their temporal and spatial distribution ${ }^{16,17}$ suggest that CFU-S from the AGM region colonize the liver.

In addition to these data on the multipotent CFU-S progenitor, Godin and colleagues found the intraembryonic para-aortic splanchnopleura (PAS) at E8.5 to contain progenitors for the Bla subset of B lymphocytes by transplantation of this embryonic region under the kidney capsule of severe combined immunodeficiency (SCID) mice. ${ }^{10}$ In a two step culture system multipotent progenitors for the B and T 
lymphoid lineages as well as erythroid-myeloid lineages were found in the PAS at E8.5. ${ }^{9}$ These cells were found to be positive for the AA4.1 surface marker previously found to be on fetal liver hematopoietic progenitors and stem cells.

With these convincing data that multipotent hematopoietic progenitors could be found in the PAS/AGM region, long-term mouse radiation chimeras were generated to test whether the AGM region contains pluripotent adult-type hematopoietic stem cells. ${ }^{21}$ Transplantations were performed as described for the CFU-S experiments, and mice were tested for engraftment at greater than 4 months posttransplantation. No donor-derived engraftment was observed for E8 or E9 yolk sac or AGM. However, transplanted E10 AGM region cells were able to fully engraft 3 out of 100 recipients, while E10 yolk sac resulted in no engraftment. Even at 8 months posttransplantation, E10 AGM region cells can repopulate the blood system of the recipients up to $100 \%$ in all hematopoietic tissues and lineages. Secondary and tertiary serial transplantations of bone marrow from these recipients indicated that the E10 AGM hematopoietic stem cells are self-renewing. Moreover, at E11 the AGM region contains a high frequency of such hematopoietic stem cells as demonstrated by the complete long-term engraftment of 11 out of 19 recipients. E11 yolk sac and liver also contained hematopoietic stem cells but at a lower frequency. Recently, Yoder and colleagues have shown that at E9 both the yolk sac and AGM region contain multipotent progenitors that can in vivo repopulate neonatal but not adult recipient mice. ${ }^{26,27}$ These data suggest that the earlier progenitors in the yolk sac and AGM have some but not all the characteristics of adult hematopoietic stem cells. Thus, the AGM region appears to be the first site within the mouse conceptus to initiate fully competent adult-type hematopoietic stem cells.

\section{THE AGM REGION AUTONOMOUSLY INITIATES HEMATOPOIETIC STEM CELL ACTIVITY}

At E10 the AGM region contains the first hematopoietic stem cells at limiting numbers. ${ }^{21}$ However, it is possible that these stem cells are generated at another site within the mouse conceptus and quickly migrate through the circulation or interstitially to localize in the AGM region. The circulation between the yolk sac and the embryo body is established at E8.5 in gestation. ${ }^{3}$ Thus, to examine the site of initiation of the first hematopoietic stem cells, we instituted an organ explant culture step for AGM, yolk sac or liver before the in vivo transplantation assay. ${ }^{15}$ Two to three days of organ culture of individual AGM, yolk sac or liver explants alleviates any cellular exchange between these tissues. When we examined E9 cultured tissues, no hematopoietic stem cells were found in AGM, yolk sac or liver. The first hematopoietic stem cells appeared in E10 cultured AGM explants. Quantitatively these stem cells outnumbered those found in uncultured AGMs by a factor of 15, strongly suggesting the induction and/or expansion of hematopoietic stem cells during the 3-day in vitro culture period. Interestingly, no hematopoietic stem cells were found in E10 yolk sac or liver but began to appear in E11 and late E11 cultured yolk sac and liver, respectively. These results show the autonomous and exclusive production of hematopoietic stem cells at E10 by the AGM region and suggest the colonization of liver by AGM-derived hematopoietic stem cells. The yolk sac may also be colonized 
by AGM-derived hematopoietic stem cells and/or endogenously generate its own hematopoietic stem cells beginning at E11.

A similar organ culture step was used to determine the site of generation of the first multipotent (lymphoid-myeloid-erythroid) progenitors. ${ }^{2}$ At E7.5 and before the circulation is established between the embryo body and the yolk sac, these progenitors are found only in the para-aortic splanchnopleura. The yolk sac contains such progenitors only after E8.5. Thus, these definitive hematopoietic progenitors as well as the first fully competent adult-type hematopoietic stem cells are autonomously generated within the embryo body before they appear in the yolk sac.

\section{THE FIRST HEMATOPOIETIC STEM CELLS ARE C-KIT ${ }^{+}$CD34 $^{+}$ AND LOCALIZE TO THE ANTERIOR AGM}

The phenotypic characterization of the AGM hematopoietic stem cells was performed. Cell surface markers indicative of adult-type hematopoietic stem cells were found on AGM hematopoietic stem cells. ${ }^{22}$ When transplanted in at various doses, E11 hematopoietic stem cells were always found in the c-kit ${ }^{+} \mathrm{CD} 34^{+}$double positive population. The E9 yolk sac and AGM cells that possess the ability to repopulate conditioned new born mice (but not adult recipients) to low levels are also c-kit ${ }^{+}$and CD $34^{+}{ }^{27}$ However, the Sca-1 hematopoietic stem cell marker, which is a marker of the hematopoietic stem cells of fetal liver ${ }^{12}$ and adult bone marrow ${ }^{23}$ but not yolk sac hematopoietc cells ${ }^{11}$ is expressed on E11 AGM hematopoietic stem cells ${ }^{8}$ suggesting this as a distinctive marker of only true definitive hematopoietic stem cells. Interestingly c-kit ${ }^{+} \mathrm{CD} 34^{+} \mathrm{E} 11 \mathrm{AGM}$ hematopoietic stem cells are negative for all mature lineage markers except Mac-1. ${ }^{22}$ While fifty percent of the AGM stem cells were found to be Mac-1 negative, the other fifty percent were Mac-1 positive and have been suggested to be the subset of AGM stem cells ready to colonize the fetal liver (at E11, E12 and E13 all fetal liver hematopoietic stem cells are Mac-1 positive). These marker studies strongly suggest a direct lineage relationship between the hematopoietic stem cells of the AGM region and the fetal liver and together with the results of the organ culture studies suggest a colonization of the fetal liver with AGM-generated hematopoietic stem cells.

Using a transgenic mouse expressing the LacZ reporter gene in Sca-1-expressing cells, we examined the localization of Sca-1-LacZ-positive cells within intact AGM tissue. ${ }^{18}$ When E10, E11, and E12 embryos were examined, beta-galactosidase-positive cells were found in the anterior portion of the AGM region. The cells lining the pronephric and mesonephric tubules stained brightly, while the cells in surrounding mesenchyme stained to an intermediate level. All yolk sac cells were negative. Moreover the kinetics of AGM beta-galactosidase staining coincided with the presence of hematopoietic stem cells in the AGM region, thus suggesting a localization for the first stem cells. By using the organ culture system we also tested whether we could further localize the first functional hematopoietic stem cells to the anterior and/or posterior portion of the AGM. ${ }^{15}$ In two experiments we found all hematopoietic stem cell activity in the anterior portion of cultured E10 AGMs. Hence the beta-galactosidase-positive cells may provide a precise localization for the first hematopoietic stem cells in the AGM region, although at this time it is undetermined 
whether the beta-galactosidase positive-cells in the mesonephric tubules or the cells within the surrounding mesenchyme (which stain less intensely) are the functional stem cells. Further functional experiments are in progress to examine which positive population contains the hematopoietic stem cells.

\section{CONCLUSIONS AND FUTURE DIRECTIONS}

The results of our in vivo transplantation experiments using the mouse as a model for mammalian hematopoiesis, clearly demonstrate the potency of the AGM region in the initiation of adult-type hematopoietic stem cells. The AGM region autonomously and exclusively inititates the first adult-type hematopoietic stem cells at E10, one day earlier than these cells can be found in the yolk sac. Previously, in contrast to the intraembryonic source found in nonmammalian vertebrates, the yolk sac was thought to be the generating source of these stem cells in mammals. The discovery

of the hematopoietic potential of the AGM region now yields a clear case for strong similarities in developmental hematopoiesis between all vertebrate species. It appears that the mouse yolk sac participates predominantly in the generation of the embryonic hematopoietic system, while the AGM region is dedicated to the initiation and production of the adult hematopoietic system.

Indeed this appears to be reflected in the genetic programming of cells destined to become embryonic or adult hematopoietic cells (reviewed in Ref. 7). Recent advances in targeted mutagenesis in mice have yielded results that demonstrate the differential requirement for some genes (AML-1, GATA-2, GATA-3, c-kit) in adult and fetal liver hematopoiesis but not embryonic hematopoiesis. In constrast the mutation of other genes (flk-1, tal-1/SCL) results in the impairment of hematopoiesis in both the yolk sac and fetal liver. Thus, the genetic programs of embryonic and adult hematopoietic cells appear to be initially overlapping during stages determining hematopoietic fate, but become unique as more complex programming is required in cells destined to become part of the adult hematopoietic system. It may be predicted that the primary cellular defects of the genes affecting fetal liver hematopoiesis occur in the induction, expansion or maintenance of hematopoietic stem cells and progenitors in the AGM region before they colonize the fetal liver. We are currently examining the roles of such genes in the AGM region and in the generation of the adult hematopoietic system by organ explant cultures and radiation chimera approaches.

Finally, the question remains how are the first fully competent adult-type hematopoietic stem cells generated in the AGM region? As the fetal thymic organ culture system has yielded great advances in knowledge concerning thymocyte differentiation and development, we hope to make use of chimeric AGM organ cultures to determine whether the precursors for the first hematopoietic stem cells are generated in situ within the AGM region or are recent emigrants from other embryonic sites such as the yolk sac. These cultures will also be used to test whether adult bone marrow hematopoietic stem cells can be increased in number in the AGM microenvironment. Recent findings of Mukouyama et al. ${ }^{20}$ suggest that indeed hematopoietic progenitors from the AGM region can be increased in vitro. Hence, our future investigation will include differential cloning to address what are the unique signals with- 
in the AGM microenvironment that lead to the induction and/or proliferation of the first fully competent adult-type hematopoietic stem cells.

\section{ACKNOWLEDGMENTS}

The author would like to thank Drs. Alexander Medvinsky, Albrecht Muller, Maria-Jose Sanchez, Colin Miles, Angus Sinclair and Marella de Bruijn for their dedication and long hours spent in the laboratory to achieve the results presented here. My thanks also to all the members of my laboratory and the Department of Cell Biology, Erasmus University who contributed to this work. This research is supported in part by the Leukemia Society of America and The Netherlands Scientific Research Organization.

\section{REFERENCES}

1. Abramson, S. et al. 1977. The identification in adult bone marrow of pluripotent and restricted stem cells of the myeloid and lymphoid systems. J. Exp. Med. 145: 15671579.

2. Cumano, A. et al. 1996. Lymphoid potential, probed before circulation in mouse, is restricted to caudal intraembryonic splanchnopleura. Cell 86: 907-916.

3. Delassus, S. \& A. Cumano. 1996. Circulation of hematopoietic progenitors in the mouse embryo. Immunity 4: 97-106.

4. Dieterlen-Lievre, F. 1975. On the origin of haemopoietic stem cells in the avian embryo: an experimental approach. J. Embryol. Exp. Morphol. 33: 607-619.

5. Dieterlen-Lievre, F. \& N.M. Le Douarin. 1993. Developmental rules in the hematopoietic and immune systems of birds: how general are they? Semin. Dev. Biol. 4: 325-332.

6. Dzierzak, E. \& A. Medvinsky. 1995. Mouse embryonic hematopoiesis. Trends Genet. 11: 359-366.

7. DzIERZAK, E. et al. 1998. Qualitative and quantitative aspects of haematopoietic cell development in the mammalian embryo. Immunol. Today 19: 228-236.

8. DZIERZAK, E. et al. 1995. Hematopoietic stem cell development in the mouse embryo. In Molecular Biology of Hemoglobin Switching. G. Stamatoyannopoulos, Ed.: 109121. Andover. Intercept.

9. Godin, I. et al. 1995. Emergence of multipotent hemopoietic cells in the yolk sac and para-aortic splanchnopleura in mouse embryos, beginning at 8.5 days postcoitus. Proc. Natl. Acad. Sci. USA 92: 773-777.

10. Godin, I.E. et al. 1993. Para-aortic splachnopleura from early mouse embryos contains Bla cell progenitors. Nature 364: 67-70.

11. Huang, H. \& R. Auerbach. 1993. Identification and characterization of hematopoietic stem cells from the yolk sac of the early mouse embryo. Proc. Natl. Acad. Sci. USA 90: 10110-10114.

12. IKUTA, K. et al. 1990. A developmental switch in thymic lymphocyte maturation potential occurs at the level of hematopoietic stem cells. Cell 62: 863-874.

13. Johnson, G. R. \& M.A. Moore. 1975. Role of stem cell migration in initiation of mouse foetal liver haemopoiesis. Nature 258: 726-728.

14. Jordan, C. T. et al. 1990. Cellular and developmental properties of fetal hematopoietic stem cells. Cell 61: 953-963.

15. Medvinsky, A. \& E. Dzierzak. 1996. Definitive hematopoiesis is autonomously initiated by the AGM region. Cell 86: 897-906. 
16. Medvinsky, A.L. et al. 1996. Development of day-8 colony-forming unit-spleen hematpoietic progenitors during early murine embyrogenesis: spatial and temporal mapping. Blood 87: 557-566.

17. Medvinsky, A. L. et al. 1993. An early pre-liver intraembryonic source of CFU-S in the developing mouse. Nature 364: 64-67.

18. Miles, C. et al. 1997. Expression of the Ly-6E.1 (Sca-1) transgene in adult hematopoietic stem cells and the developing mouse embryo. Development 124: 537-547.

19. Moore, M. A. \& D. Metcalf. 1970. Ontogeny of the haemopoietic system: yolk sac origin of in vivo and in vitro colony forming cells in the developing mouse embryo. Br. J. Haematol. 18: 279-296.

20. Mukouyama, Y. et al. 1998. In vitro expansion of murine hematopoietic progenitors from the embryonic aorta-gonad-mesonephros region. Immunity 8: 105-114.

21. Muller, A. M. et al. 1994. Development of hematopoietic stem cell activity in the mouse embryo. Immunity 1: 291-301.

22. SANCHEZ, M. J. et al. 1996. Characterization of the first definitive hematopoietic stem cells in the AGM and liver of the mouse embryo. Immunity 5: 513-525.

23. SPANGRUde, S.J. et al. 1988. Purificaiton and characterization of mouse heamtopoietic stem cells. Science 241: 58-62.

24. TURPEN, J. B. et al. 1997. Bipotential primitive-definitive hematopoietic progenitors in the vertebrate embryo. Immunity 7: 325-334.

25. Turpen, J. B. et al. 1981. The early ontogeny of hematopoietic cells studied by grafting cytogenetically labeled tissue anlagen: localization of a prospective stem cell compartment. Dev. Biol. 85: 99-112.

26. Yoder, M.C. \& K. HiatT. 1997. Engraftment of embryonic hematopoietic cells in conditioned newborn recipients. Blood 89: 2176-2183.

27. Yoder, M.C. et al. 1997. Characterization of definitive lymphohematopoietic stem cells in the day 9 murine yolk sac. Immunity 7: 335-344.

\section{DISCUSSION}

G. Keller (Howard Hughes Medical Institute): A technical question regarding your reaggregation experiments with the bone marrow stem cells. Were the reaggregated organs cultured?

DZIERZAK: The reaggregation culture is for 4 days.

KELLER: Could you speculate as to why cells with long-term repopulating potential would move from the AGM to the yolk sac?

DZIERZAK: In the avian system one finds movement of cells from the interbody region out to the yolk sac in the grafting experiments, so there is a precedent for it. Just by analogy I would think this probably does happen in the mouse, but we need to have direct evidence for these things. One really needs to in situ dye-mark those cells and see whether they actually go out. We would like to do these experiments. Time will tell.

S.J. SHAR KIS (Johns Hopkins Oncology Center): If you take day 11 embryos and then put them in culture for only 1 day, so that it is at least equivalent in terms of time to your 10-day embryos that are in culture for two days, do you see an equivalent increase in progenitors that you would see otherwise?

DZIERZAK: We are trying to do some more quantitative experiments, and it requires a lot of competitive repopulation or limiting dilution transplantation analysis. 
It is going to be difficult to be absolutely sure about how the in vivo data compare with this in vitro situation, because in the in vitro situation the cells do not move out. They can be generated there but not move out. So we have an accumulation of these cells, whereas in the embryo itself the cells are rapidly moving out as soon as they are produced to other places. So it is going to be very hard to be absolutely sure about how the cells are growing and their numbers in the culture versus in vivo.

SHARKIS: But in vivo you are actually seeing an increase at day 11 above day 10 in vivo.

DZIERZAK: Yes.

ShARKIS: So one would think that you could actually tell quantatively.

DZIERZAK: Yes, I think the production of these cells increases at day 11 in the AGM region, but we have to really measure that carefully.

T. PAPAY YNNOPOULOU (University of Washington): In your reaggregation experiments, in which you mixed bone marrow cells with the AGM, you elected to use only the $\mathrm{CD} 31^{+} / \mathrm{Ly}-6^{+} / \mathrm{kit}^{+}$cells. Why?

DZIERZAK: At Erasmus University the post doc M. de Bruijn has previously done sorting experiments for stem cells with Rob Ploemacher. They have developed these ERMP12/20 antibodies that they have used for many such sorting experiments. This was just the easiest way to get at an enriched population, because we did not want to use the whole bone marrow. There are too many cells, and the frequency of the stem cells is too limited. We wanted some fractionation procedure, so we just used the one that was close at hand.

G.J. SPAngRUde (University of Utah Medical Center): One hypothesis that might explain the difference between your transplants into adult recipients compared to the transplants into perinatal or embryonic recipients is that you are actually following the emergence of what I am considering to be the "bank account" of stem cells that corresponds to the back-up source of reconstituting cells. So during the early course of embryogenesis, most of these cells are in an expanding phase, because they need to generate the hematopoietic system. But then you could say that around day 10 some of them start to go into a more quiescent compartment that eventually leads to the build-up of the back-up compartment. Along those lines I am wondering if you have looked at cell cycle analysis. Can you tell us whether these cells when you isolate them are actively cycling cells and if this correlates, as we see in adults, with engraftment by noncycling cells?

DzIERZAK: We are trying to look at this right now. It is very difficult for us to do it for numerous technical reasons. For the first look (and this is really preliminary, but you would expect this from embryonic cells), they are all cycling. But that is very preliminary, and we have to do a lot of engrafting experiments to be sure.

SPANGRUde: So it does not really bother me if they are all cycling. Maybe the difference is time spent in G1 between cycles, and this is what allows these cells to engraft.

DzIERZAK: Yes, we do not know. We have to look very carefully at this question; it is very important.

D. Metcalf (P.O. Royal Melbourne Hospital): There is a lot to be said for "seeing is believing." If one cultures a whole embryo with an intact yolk sac, it develops a heart that beats and that is pumping around blood cells. If one cultures the embryo alone, it develops a heart that pumps around cell free fluid. So I would like to know, 
in this sort of experiment what has happened to the function of the AGM, because the latter animal totally lacks hematopoietic cells? In relation to your organ culture experiments, can you be sure that the organ tissue survival and proliferation is equivalent in each case? I noticed, for example, that your liver cultures did not develop any repopulating cells. This is slightly odd, and liver is a difficult tissue to culture en masse. I think your chimeric organ cultures will approach this. I hope you use a combination of marked cells so that you can see whether with improved culture conditions, the yolk sac can generate repopulating cells.

DZIERZAK: Yes, we are using marked cells and, in combination in these chimeric organ cultures, we would like to use AGM region cells from knock-out embryos. We are testing to see whether they are deficient, say, in hematopoitic stem cells or stromal cells or whatever. The culture conditions for the liver certainly are not optimal. We would like to make them optimal. Late day-11 liver does give us hematopoietic stem cell activity.

As for the yolk sac culture experiment, we want to revisit that experiment, the one that you did long ago. It is a very provocative experiment and an interesting one. Yes, perhaps one needs a yolk sac not only very rapidly to produce red cells to oxygenate the tissues, but perhaps to help the heart in some form of development or setting up the liver to be accepting later-stage, developmentally more mature hematopoietic cells. I am curious whether the AGM region in such an animal is going to be functional with or without the AGM region. We would like now to do whole embryo cultures, and we are trying to establish that in the laboratory. It is a very good and interesting way to go about some of studies, and it may in fact give us many more answers than the individual organ explant culture will. 